Journal of

\section{Applied Crystallography}

ISSN 0021-8898

Received 7 August 2008

Accepted 21 October 2008

\title{
Combining precession electron diffraction data with X-ray powder diffraction data to facilitate structure solution
}

\author{
Dan Xie, Christian Baerlocher and Lynne B. McCusker* \\ Laboratory of Crystallography, ETH Zurich, Switzerland. Correspondence e-mail: \\ mccusker@mat.ethz.ch
}

\begin{abstract}
Information derived from precession electron diffraction (PED) patterns can be used to advantage in combination with high-resolution X-ray powder diffraction data to solve crystal structures that resist solution from X-ray data alone. PED data have been exploited in two different ways for this purpose: (1) to identify weak reflections and (2) to estimate the phases of the reflections in the projection. The former is used to improve the partitioning of the reflection intensities within an overlap group and the latter to provide some starting phases for structure determination. The information was incorporated into a powder charge-flipping algorithm for structure solution. The approaches were first developed using data for the moderately complex zeolite ZSM-5, and then tested on TNU-9, one of the two most complex zeolites known. In both cases, including PED data from just a few projections facilitated structure solution significantly.
\end{abstract}

\section{Introduction}

Over the years, a broad palette of methods for determining the crystal structure of a polycrystalline material from X-ray powder diffraction (XPD) data has been developed, and it is now possible to solve moderately complex structures in an almost routine manner [see the review by David \& Shankland (2008), and references therein]. Recently, it was shown that even more complex structures can be addressed, if phase information derived from high-resolution transmission electron microscopy (HRTEM) images is introduced into the structure determination procedure (Gramm et al., 2006; Baerlocher, Gramm et al., 2007; Baerlocher et al., 2008). In light of these results, the feasibility of using another, less demanding, electron microscopy technique, namely precession electron diffraction (PED), to augment the X-ray powder diffraction data has been explored.

The key problem in structure determination from powder diffraction data arises from the fact that reflections with similar $2 \theta$ values, though unrelated from a structural point of view, overlap in the powder pattern. As a result, their individual intensities are ambiguous, and this hinders structure solution. All methods of structure determination would benefit if this ambiguity could be resolved, at least in part. Two experimental approaches to this problem, both involving the collection of multiple X-ray powder diffraction data sets on the same sample under different conditions, have already been reported (Wessels et al., 2002). Both approaches, one exploiting anisotropic thermal expansion and the other preferred orientation, have been shown to be effective, but both involve non-routine data collections and data analyses.
Here we consider the viability of using one or more electron diffraction patterns to supplement a single high-resolution $\mathrm{X}$-ray powder diffraction pattern.

Electron and X-ray powder diffraction are remarkably complementary techniques (see Table 1). Electrons interact much more strongly with matter than do X-rays, so a singlecrystal electron diffraction pattern can be obtained from a very tiny crystallite. Such patterns have two obvious advantages: (1) the pattern is that of a single crystal, so there is no reflection overlap, and (2) the interaction is stronger, so the resolution in $d$ spacing is higher. However, the strong interaction generally results in complicated multiple scattering effects, which cause the reflection intensities to deviate severely from the kinematical assumption that $I_{h k l}$ is proportional to $\left|F_{h k l}\right|^{2}$ (Cowley, 1995). This is the main reason that electron diffraction data are not generally used for structure determination, although there are some notable exceptions in the literature (e.g. Dorset, 1995; Zou \& Hovmöller, 2008).

Almost 15 years ago, Vincent \& Midgley (1994) introduced the precession electron diffraction technique as a partial solution to this problem. This technique is equivalent to the Buerger precession method used in single-crystal X-ray diffraction, where the crystal is precessed around the incident $\mathrm{X}$-ray beam. In the PED case, however, the electron beam is deflected and precessed around a stationary crystal. With this tilted illumination, only a few reflections are excited simultaneously, so the possibilities for multiple scattering are reduced and the resulting diffraction intensities are more kinematical in nature. Now that commercial PED attachments for existing electron microscopes have become available, this promising technique has become accessible to a much broader community. 
Table 1

Complementarity of X-ray powder and electron diffraction.

\begin{tabular}{lll}
\hline & X-ray powder diffraction & Electron diffraction \\
\hline Crystallite size & $\mu \mathrm{m}$ & $\mathrm{nm}$ \\
Data set & Complete & Incomplete \\
Resolution in $d$ & Low & High \\
Data precision & High & Low \\
Intensities & Kinematical (easy to & Dynamical (difficult to \\
& interpret) & interpret) \\
Multiple scattering & No & Yes \\
Overlap & Yes & No \\
\hline
\end{tabular}

Although the PED reflection intensities are more reliable than those from a selected area electron diffraction (SAED) pattern, they are still not ideal. Own et al. (2006) have shown that dynamical effects still occur in PED patterns, with the high- $d$-spacing (low-angle) reflections being most affected. As might be expected, the precession angle and crystallite thickness proved to be critical parameters. Nonetheless, we reasoned that the PED patterns should contain information that could be used to advantage in combination with X-ray powder diffraction data.

Two possibilities for combining the information from the two sources were investigated. In one case, the PED data were simply used to identify the weak reflections in selected projections, with the hope that this information would lead to a more correct partitioning of some of the overlapping reflections in the powder pattern. In the second case, the single-crystal charge-flipping structure solution algorithm of Oszlányi \& Sütô $(2004,2005,2008)$ was applied to the twodimensional PED data for selected zones to obtain phases for the contributing reflections. These phases were then included in the starting phase sets for the application of the powder charge-flipping $(p C F)$ algorithm (Baerlocher, McCusker \& Palatinus, 2007) to the X-ray powder diffraction data. This approach resembles that used previously for the combination of X-ray powder diffraction data with phases derived from HRTEM images.

To develop the two approaches, both simulated and experimental data for the moderately complex zeolite ZSM-5 (van Koningsveld et al., 1987; Pnma; $a=20.022, b=19.899, c=$ $13.383 \AA$, 38 atoms in the asymmetric unit, 288 atoms in the unit cell) were used. The methods were then tested using experimental data for the very complex zeolite TNU-9 (Gramm et al., 2006; $C 2 / m ; a=28.222, b=20.012, c=19.493 \AA$, 76 atoms in the asymmetric unit, 576 atoms in the unit cell).

\section{Experimental}

Samples of as-synthesized ZSM-5 and calcined TNU-9 were used for the powder diffraction data collections. For the PED measurements, both samples were calcined, crushed using a mortar and pestle, suspended in ethanol, and disaggregated with ultrasound. Structure factors for the simulations were calculated using the structural data published by van Koningsveld et al. (1987) for ZSM-5 and by Gramm et al. (2006) for TNU-9.
The experimental PED data were collected at $300 \mathrm{kV}(\lambda=$ $0.0197 \AA$ ) on a Philips CM30 electron microscope equipped with a 'Spinning Star' precession unit (NanoMegas) and a 16 bit Gatan 794 CCD camera. Exposure times were selected such that the diffraction intensities lay within the linear response range of the detector. The patterns were recorded with a precession angle close to $1^{\circ}$ and the intensities were extracted using the program ELD (Zou et al., 1993) in the CRISP software package (Hovmöller, 1992). Phases from PED projections were derived using the single-crystal chargeflipping algorithm in the program Superflip (Palatinus \& Chapuis, 2007).

The high-resolution synchrotron powder diffraction data for ZSM-5 were collected on the Swiss Norwegian Beamline (SNBL) at the ESRF in Grenoble $(\lambda=1.1011 \AA)$ and those for TNU-9 at the SRS in Daresbury ( $\lambda=0.99556 \AA)$. The intensity extractions were performed using the program EXTRACT (Baerlocher, 1990) in the $X R S$-82 suite of programs (Baerlocher \& Hepp, 1982).

The powder charge-flipping algorithm implemented in the program Superflip was used for structure solution (Baerlocher, McCusker \& Palatinus, 2007). In this implementation, the intensity repartitioning procedure for overlapping reflections is coupled to a second modification of the electron density map based on histogram matching. The reference histogram used for this step simply reflects the chemical composition of the material.

\section{Weak reflection elimination}

Although it was hoped initially that all the PED data could be used directly to supplement the XPD data, this proved not to be possible, because (1) the PED intensities are still distorted from the kinematical case, and (2) the atomic scattering factors (and therefore the structure factors) for X-rays and electrons are not strictly proportional to one another (see Fig. 1). However, the scattering factors do show the same general trend. Dorset et al. (1998) exploited this similarity ten years ago to scale a set of SAED patterns to one another using the X-ray powder diffraction pattern as a reference. In our

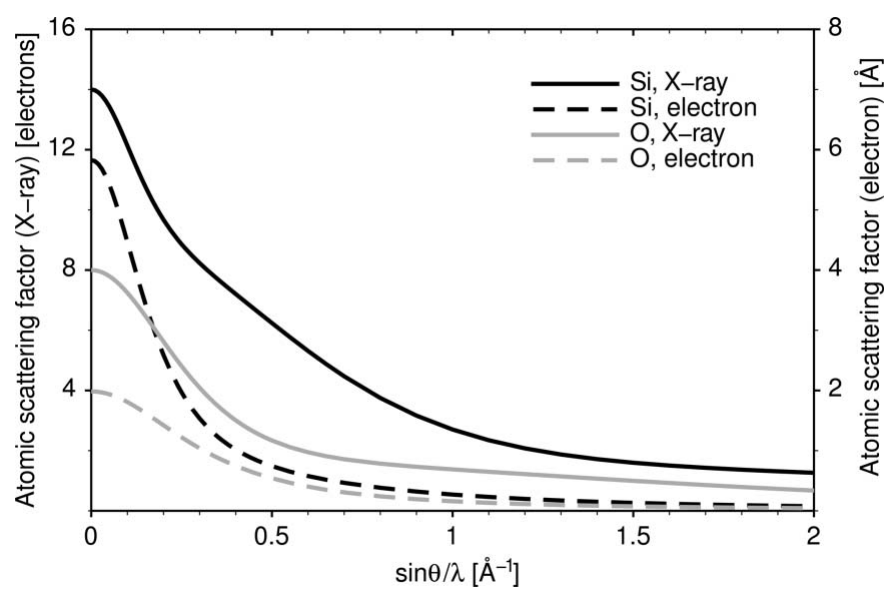

Figure 1

Atomic scattering factors of $\mathrm{Si}$ and $\mathrm{O}$ for $\mathrm{X}$-rays and electrons. 
case, it was reasoned that reflections that are weak in the PED pattern should also be weak in the X-ray diffraction pattern.

A very simple and conservative procedure was applied to take advantage of this information. The PED data were used to identify the weak reflections in a projection, and then these reflections were eliminated from the XPD intensity extraction. Generally, the intensity ratios of overlapping reflections are set to one (equipartitioning), so weak reflections in an overlap group can be assigned medium or even large structure-factor amplitudes. It was hoped that, by eliminating these weak

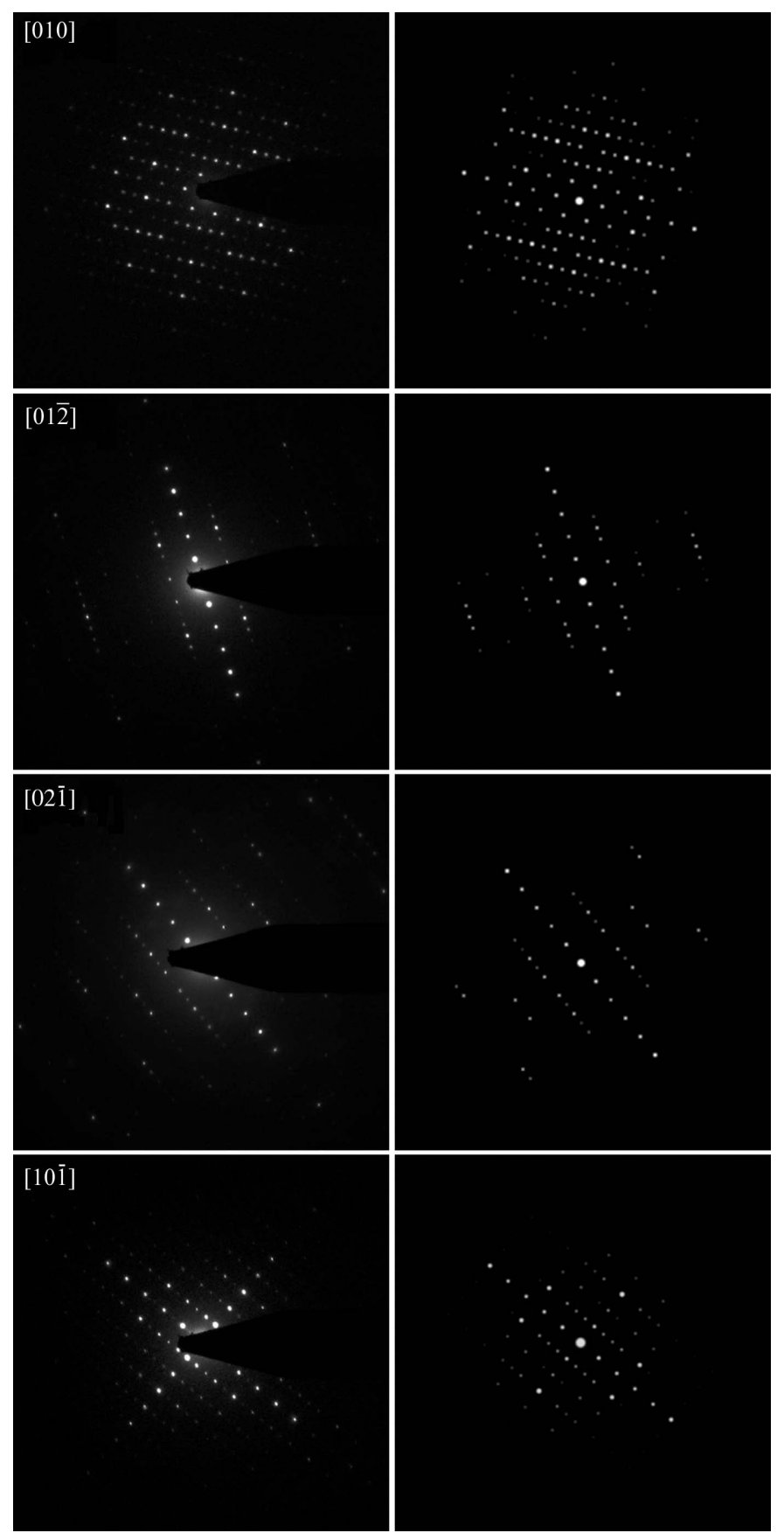

Figure 2

Precession electron diffraction patterns for ZSM-5 (left) and their

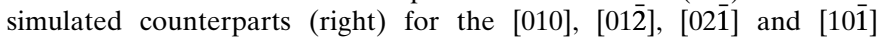
projections. reflections from consideration, a more correct partitioning of the remaining reflections in the group would be obtained.

Initial tests of this weak reflection elimination (WRE) approach were performed on the zeolite ZSM-5 using both simulated and experimental PED patterns along four zone axes ([010], [012],$[02 \overline{1}]$ and [101]]). The crystallite thicknesses for the PED measurements were estimated to be less than $100 \mathrm{~nm}$. For simplicity, the structure-factor amplitudes were calculated using the kinematical assumption, and no geometrical corrections were applied. The PED amplitudes were scaled to the simulated ones by applying a least-squares fit to the data. Although the experimental patterns look qualitatively similar to the simulated ones (Fig. 2), there is considerable scatter in the amplitudes (Fig. 3). For the data shown in Fig. 3, for example, the $R$ value for the agreement between the two is only 0.51 . Similar scatter is observed in ten other [010] patterns collected over a period of time under different conditions. Data collected with a precession angle of $1.2^{\circ}$ appear to be slightly better $(0.51 \leq R \leq 0.53)$ than those collected with a precession angle of $0.8^{\circ}(0.55 \leq R \leq 0.57)$. The reproducibility is also better with the higher precession angle $\left(0.15 \leq R_{\text {merge }} \leq 0.17\right.$ at $1.2^{\circ}$ versus $0.19 \leq R_{\text {merge }} \leq 0.22$ at $\left.0.8^{\circ}\right)$.

In order to identify which reflections should be considered to be weak, an amplitude threshold had to be defined. It is important to note that, unlike the simulated data, the experimental PED intensities derived from different projections are not on the same scale, because they are taken with different illumination conditions, exposure times and crystal thicknesses (Gemmi et al., 2002), so it is not sensible to set the same threshold value for all four data sets. Attempts to scale the patterns to one another using common reflections were not very satisfactory, so it was considered to be better to treat each

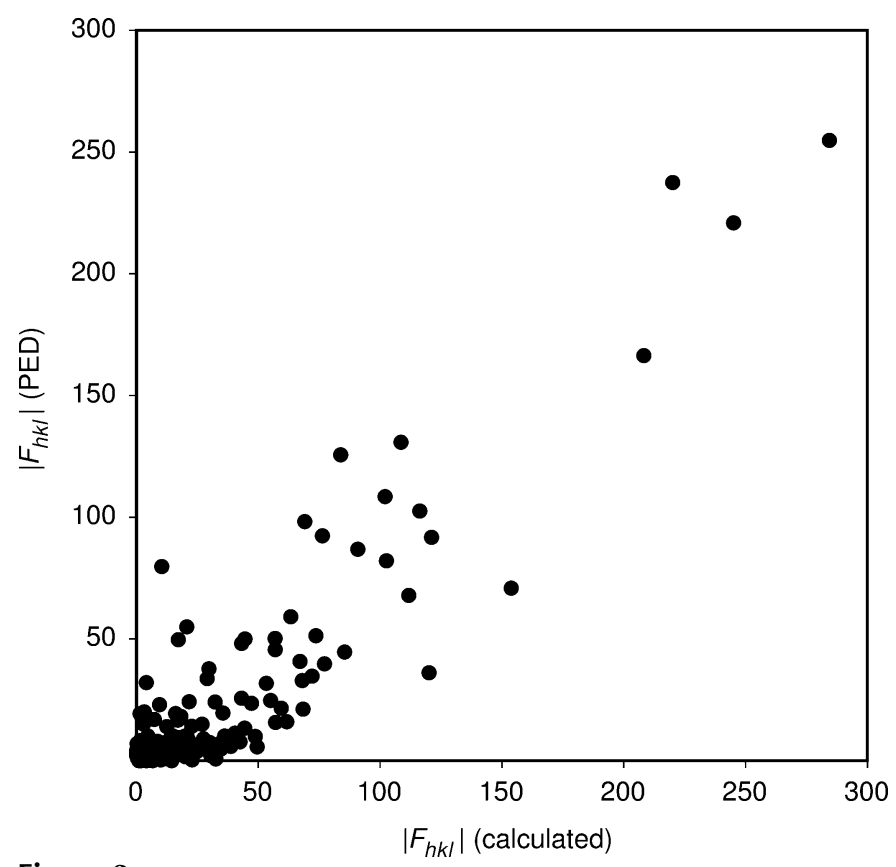

Figure 3

Comparison between experimental (PED) and calculated structurefactor amplitudes for the [010] projection of ZSM-5. 
projection independently. The threshold for each projection was chosen for both the simulated and the experimental data by examining a plot of the amplitude distribution (Fig. 4) and selecting a value such that the reflections to be eliminated were in the range of the tail of the profile. To evaluate how sensitive the structure solution was to the choice of this threshold, ten values ranging from 10 to $100 \%$ of the average amplitude were tested. For each threshold, the reflections defined to be weak were eliminated from the $h k l$ list and the remaining reflection intensities were re-extracted. Each of the 20 modified data sets (ten simulated and ten experimental) were then used as input for 100 runs of the powder chargeflipping algorithm $(p C F)$ in the program Superflip (Palatinus \& Chapuis, 2007).

The $p C F$ solutions in Superflip are ranked according to an $R$ value that compares the experimentally measured structurefactor amplitudes $\left(\left|F_{h k l}\right|\right)$ with those derived from the chargeflipped map $\left(\left|G_{h k l}\right|\right)$ :

$$
\sum_{h k l}|| G_{h k l}|-| F_{h k l}|| / \sum_{h k l}\left|F_{h k l}\right| .
$$

To evaluate the correctness of the electron density maps produced in the $p C F$ runs, the ten best maps according to this Superflip $R$ value were compared with a reference map calculated from the true structure, and an average agreement factor $R_{\text {map }}$ was calculated (Fig. 5). The correctness of the maps was found to be substantially better for threshold values within the $30-80 \%$ range and relatively stable within this range. It is apparent from these comparisons that the WRE method can improve the electron density maps produced by Superflip significantly. In contrast to $p C F$ electron density maps generated without WRE, those generated with WRE (30-80\% range) revealed the positions of all $12 \mathrm{Si}$ and $26 \mathrm{O}$ atoms in the asymmetric unit clearly. It appears that even this simple modification of the data set has a significant impact on the structure solution.

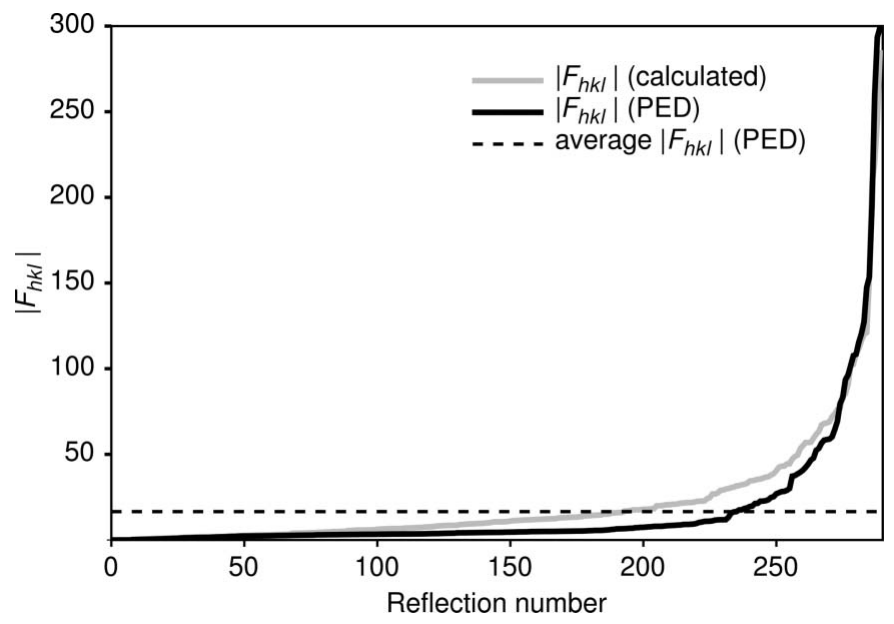

Figure 4

Experimental (PED) and calculated structure-factor amplitudes for the [010] projection arranged in ascending order. The average $\left|F_{h k l}\right|$ value for the PED data that was used to define the weak reflections is indicated with a dashed line. Plots for the other three projections shown in Fig. 2 are similar.

\section{Phase retrieval from precession electron diffraction data}

Precession electron diffraction data have already been used in combination with direct methods (Weirich et al., 2006) and maximum entropy methods (Dorset et al., 2007; Gilmore et al., $2008 a, b)$ to determine two-dimensional structures. This is an indication that the reflection intensities are reliable enough and have sufficient resolution for the phasing process to succeed. For three-dimensional structures, of course, a single projection is insufficient to solve the structure, but it was reasoned that, if the phases for selected projections could be determined from PED data, these phases could be used in combination with X-ray powder diffraction data in the same way as those derived from electron microscopy images. In this study, the charge-flipping algorithm of Oszlányi \& Sütő (2004, 2005) rather than direct methods or maximum entropy methods was used for the phase retrieval step.

The simulated and experimental electron diffraction data for ZSM-5 that were used to develop the weak reflection elimination approach were also used for these tests. The phase retrieval procedure was extremely simple and automatic. 100 charge-flipping runs, each with 500 iterations, were performed on each of four two-dimensional data sets (289 reflections for the [010] zone, 162 reflections for [012], 124 reflections for

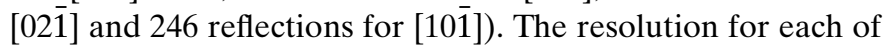
these data sets was $c a 0.8 \AA$. For each projection, the five maps with the best Superflip $R$ value were averaged. Then a Fourier transform was applied to calculate the phases of the corresponding reflections. Even though the symmetry of the map at this stage was $P 1$, the space-group symmetry was imposed for this transformation to ensure that only phases consistent with a centrosymmetric space group were obtained. A comparison of these phases with the correct ones is given in Table 2.

It is readily apparent from Table 2 that the phases of the stronger reflections are more likely to be correct than those of the weaker reflections. If the total amplitude that is correctly

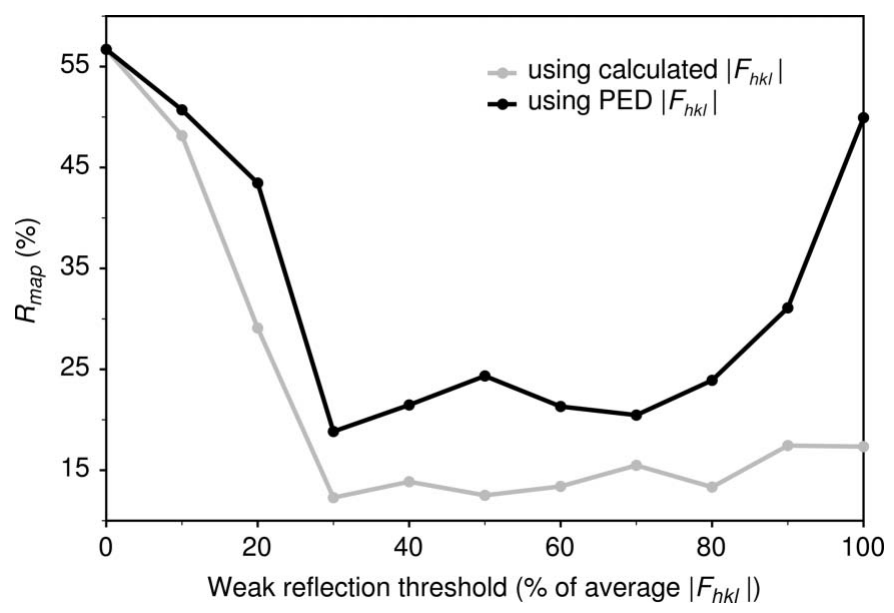

Figure 5

Effect of the weak reflection threshold value ( $\%$ of the average $\left|F_{h k l}\right|$ ) on the correctness of the electron density maps $\left(R_{\mathrm{map}}\right)$ generated by $p C F$. The point for each threshold value reflects the average $R_{\text {map }}$ for the ten best $p C F$ maps (lowest Superflip $R$ values). 
Table 2

Number of correct phases derived from the charge-flipping runs on electron diffraction data for ZSM-5.

\begin{tabular}{|c|c|c|c|c|c|c|c|c|}
\hline \multirow[b]{3}{*}{ Amplitudes } & \multicolumn{8}{|c|}{ Number of correct phases/number of reflections } \\
\hline & \multicolumn{2}{|l|}{ [010] zone } & \multicolumn{2}{|l|}{ [012̄] zone } & \multicolumn{2}{|l|}{$[02 \overline{1}]$ zone } & \multicolumn{2}{|l|}{$[10 \overline{1}]$ zone } \\
\hline & Simulated & PED & Simulated & PED & Simulated & PED & Simulated & PED \\
\hline$>50$ & $26 / 31$ & $23 / 31$ & $7 / 9$ & $7 / 9$ & $7 / 9$ & $7 / 9$ & $12 / 13$ & $12 / 13$ \\
\hline$>10$ & $120 / 149$ & 98/149 & $34 / 47$ & $34 / 47$ & $23 / 37$ & $24 / 37$ & $45 / 58$ & $43 / 58$ \\
\hline$>5$ & $154 / 203$ & $133 / 203$ & $47 / 69$ & $46 / 69$ & $33 / 56$ & $33 / 56$ & $66 / 94$ & $64 / 94$ \\
\hline \multirow[t]{3}{*}{$>0$} & $191 / 289$ & $166 / 289$ & $96 / 162$ & $90 / 162$ & $72 / 124$ & $75 / 124$ & $145 / 246$ & $131 / 246$ \\
\hline & \multicolumn{8}{|c|}{$\sum$ amplitudes with correct phases $/ \sum$ all amplitudes } \\
\hline & $83 \%$ & $73 \%$ & $74 \%$ & $74 \%$ & $72 \%$ & $72 \%$ & $82 \%$ & $81 \%$ \\
\hline
\end{tabular}

Table 3

Comparison of $p C F$ runs using both weak reflection elimination and phase information for ZSM-5.

\begin{tabular}{lrrr}
\hline & $\begin{array}{l}\text { Number of } \\
\text { weak } \\
\text { reflections } \\
\text { eliminated }\end{array}$ & $\begin{array}{l}\text { Number of } \\
\text { phases } \\
\text { supplied }\end{array}$ & $R_{\text {map }}(\%)$ \\
\hline PED data used & 0 & 0 & 56.7 \\
None & 136 & 79 & 34.2 \\
{$[010]$} & 227 & 109 & 33.9 \\
{$[010]+[01 \overline{2}]$} & 292 & 138 & 24.7 \\
{$[010]+[01 \overline{2}]+[02 \overline{1}]$} & 412 & 182 & 16.7 \\
\hline 010$]+[01 \overline{2}]+[02 \overline{1}]+[10 \overline{1}]$ & & &
\end{tabular}

phased is considered, this is more than $70 \%$ for all four zones, even for the experimental data. These numbers are similar to those obtained for phases derived from HRTEM images. The potential map generated from the PED amplitudes and the charge-flipping phases for the [010] projection of ZSM-5 is shown in Fig. 6. It can be seen from the projection of the structure that is overlaid that the major features are reproduced in the potential map. Thus, it would appear that the phases from PED projections might be as useful as those from an HRTEM image.

To test this, the 594 phases derived from the four PED charge-flipping runs were included in each starting phase set of 3042 reflections for $p C F$ runs using the XPD data. All phases were allowed to change during the procedure. $100 \mathrm{pCF}$ runs of 500 iterations were performed. As might be expected, the additional phase information resulted in electron density maps much closer to the true one. The final $R_{\text {map }}$ values dropped from $56.7 \%$ with just random phases in the starting phase set to $18.0 \%$ with the inclusion of the phases derived from the PED data.

\section{Combination of the two methods}

To evaluate the effect of combining the weak reflection elimination and phase retrieval approaches, a series of tests using one to four PED data sets were performed. The weak reflection threshold was simply chosen to be half the average amplitude. The results of these $p C F$ runs are summarized in Table 3. It is clear from the electron density maps generated with and without the PED data that the PED data contribute significantly to the correctness of the map. Not only are all the atoms visible in the map, but their electron density ratios also better reflect the different sizes of $\mathrm{Si}$ and $\mathrm{O}$, so (automatic) interpretation becomes easier.

\section{TNU-9}

The structure of TNU-9 $\left(\left[\mathrm{Si}_{192} \mathrm{O}_{384}\right]\right)$ is one of the two most complex zeolite structures known (Gramm et al., 2006). It was originally solved by combining high-resolution XPD data with phases derived from HRTEM images in the zeolite-specific structuresolution program FOCUS (Grosse-Kunstleve et al., 1997). For structure solution, 258 phases were input to FOCUS, and after 16 days of computing time, the correct model with $24 \mathrm{Si}$ atoms in the asymmetric unit was found. To test the methods described in the previous sections, this extremely complex structure was re-investigated using the $p C F$ algorithm supplemented with information derived from PED data.

Initial attempts to solve the structure using XPD data alone were not successful. The electron density maps with the best Superflip $R$ values showed neither clear pores nor framework atom positions (Fig. 7a). Comparison of this map with the correct one yields an $R_{\text {map }}$ value of $78.8 \%$. PED data were then used for weak reflection elimination and phase retrieval. For each PED projection, a plot of the amplitude distribution was examined to evaluate the appropriate amplitude threshold for WRE, and in each case, a value of half of the average amplitude was chosen. Using the procedure described in $\S \S 3$ and 4, weak reflections were eliminated from the XPD $h k l$ list and the intensities of the remaining reflections reextracted, and phases were derived from different PED projections using the single-crystal algorithm in Superflip.

A series of $p C F$ tests combining these data showed that the structure could be solved when five PED patterns ([001], [100], [102], [101] and [201] projections) were used. With these five

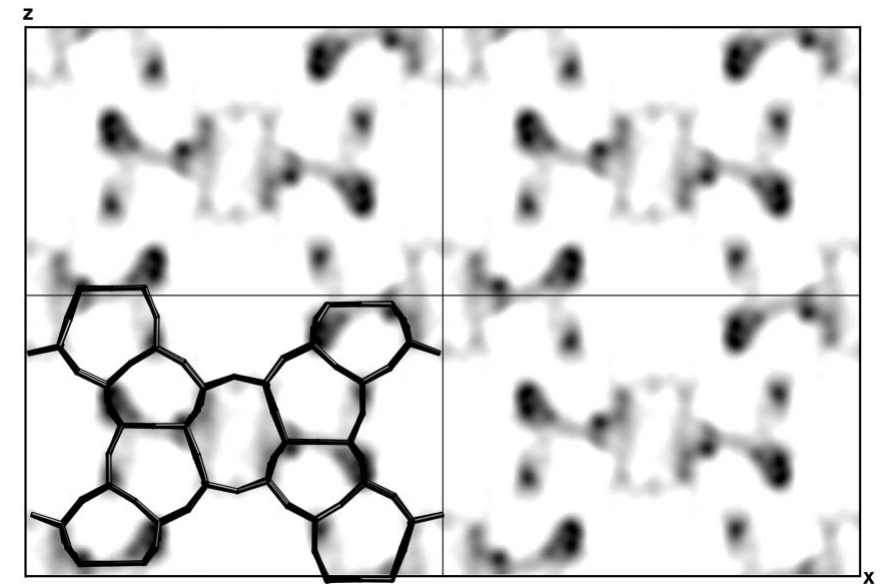

Figure 6

Two-dimensional potential map for the [010] projection generated from the PED structure-factor amplitudes and charge-flipping phases $(0.8 \AA$ resolution). The corresponding framework structure model is overlaid in the lower left hand unit cell for comparison. 
patterns, 323 weak reflections could be eliminated and 176 phases derived. Then, $100 p C F$ runs of 600 iterations each

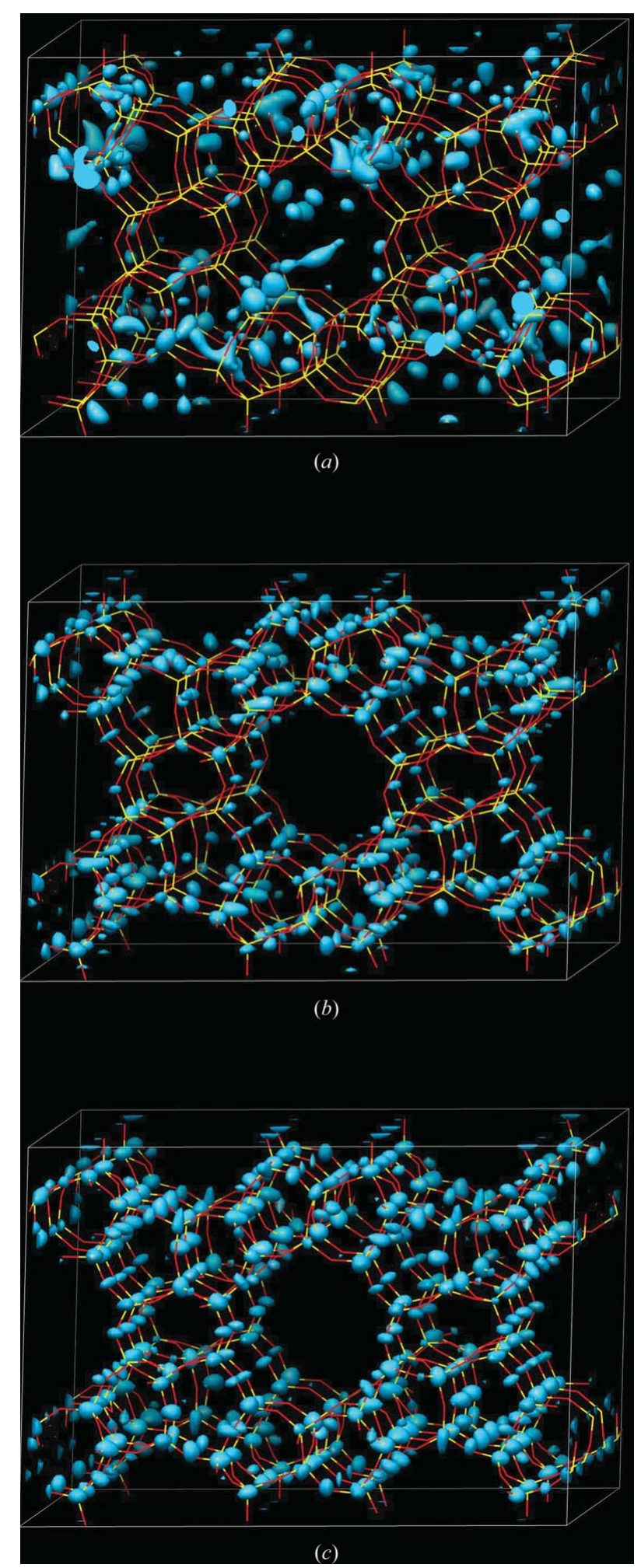

Figure 7

Powder charge-flipping electron density maps for TNU-9 generated (a) using XPD data alone, $(b)$ using XPD data and five PED patterns (combined weak reflection elimination and phase retrieval), and (c) using the map in $(b)$ as a seed for a second $p C F$ run. The framework structure of calcined TNU-9 is overlaid for comparison (Si: yellow; O: red). were performed. The input phases were enforced for the first 50 iterations and were then allowed to change like the rest. The electron density maps with the best Superflip $R$ values showed significant improvement over those generated using the XPD data alone. The peaks in the electron density maps were more spherical and the main features of the pore system appeared (Fig. $7 b, R_{\text {map }}=51.3 \%$ ). Although the best map showed the positions of 23 of the $24 \mathrm{Si}$ atoms in the asymmetric unit and many of the $\mathrm{O}$ atoms, the height of the peaks did not reflect the relative scattering powers of $\mathrm{Si}$ and $\mathrm{O}$. Therefore, this map was used as a seed in Superflip to generate 100 new starting phase sets by allowing the phases calculated from the seed map to vary by up to $20 \%$ in a random fashion. The best electron density maps resulting from this second series of $p C F$ runs showed all $24 \mathrm{Si}$ atom positions and more realistic electron densities for $\mathrm{Si}$ and $\mathrm{O}$ (Fig. $7 c, R_{\text {map }}=39.2 \%$ ).

Although the solution of the structure of TNU-9 was not quite as simple as that of ZSM-5, the additional information from the PED data did make structure solution possible. The increased difficulty can probably be attributed to three factors: (1) the complexity of the structure (twice as many atoms in the unit cell), (2) the degree of reflection overlap (92.6\% for TNU-9 versus $87.9 \%$ for ZSM-5) and (3) the lower resolution of the X-ray data (1.15 $\AA$ for TNU-9 versus $0.99 \AA$ for ZSM-5). The charge-flipping algorithm is known to be particularly sensitive to the resolution of the data. Nonetheless, the structure could be solved in a relatively straightforward manner.

\section{Conclusions}

By supplementing X-ray powder diffraction data with precession electron diffraction data, it is possible to facilitate the solution of the crystal structures of polycrystalline materials. Such PED data are considerably easier to obtain than high-resolution electron microscopy images and appear to contain similar information. PED data can be used to identify weak reflections in the X-ray powder diffraction pattern and thereby improve the reflection intensity extraction, and/or to obtain phase information for those reflections in the projection. The amount of correct phase information retrieved by applying a simple charge-flipping procedure to the two-dimensional single-crystal PED data is comparable to that that can be derived from an HRTEM image. In this initial investigation, we wanted to keep things as simple as possible, so no corrections were applied to the PED data. However, studies evaluating the effect of applying different corrections to the data are in progress.

In this paper, we have discussed the application of these two approaches to two zeolite structures of different complexities using the powder charge-flipping algorithm in the program Superflip. However, the methods are generally applicable (i.e. not zeolite specific), so it should be possible to apply them to any polycrystalline material. Furthermore, introducing information from PED data to the structure solution procedure is not restricted to charge flipping. It could also be used to advantage in other programs (e.g. in the form of better 
intensities in a direct-space global-optimization program or as starting phases for direct methods trials). The precession electron diffraction technique offers a relatively simple route to valuable information that can be used to complement that in a powder diffraction pattern.

We thank the beamline scientists of the SNBL at ESRF and at the SRS for their assistance with the powder diffraction measurements. We also thank Lukas Palatinus for the program Superflip and for his continuing close collaboration on the powder diffraction aspects of that program. Funding from the Swiss National Science Foundation is gratefully acknowledged.

\section{References}

Baerlocher, Ch. (1990). EXTRACT. Institut für Kristallographie, ETH, Zürich, Switzerland.

Baerlocher, Ch., Gramm, F., Massueger, L., McCusker, L. B., He, Z. B., Hovmöller, S. \& Zou, X. D. (2007). Science, 315, 1113-1116.

Baerlocher, Ch. \& Hepp, A. (1982). XRS-82. Institut für Kristallographie, ETH, Zürich, Switzerland.

Baerlocher, Ch., McCusker, L. B. \& Palatinus, L. (2007). Z. Kristallogr. 222, 47-53.

Baerlocher, Ch., Xie, D., McCusker, L. B., Hwang, S. J., Chan, I. Y., Ong, K., Burton, A. W. \& Zones, S. I. (2008). Nat. Mater. 7, 631-635.

Cowley, J. M. (1995). Diffraction Physics, 3rd ed. Amsterdam: Elsevier Science.

David, W. I. F. \& Shankland, K. (2008). Acta Cryst. A64, 52-64.

Dorset, D. L. (1995). Structural Electron Crystallography. New York: Plenum Press.
Dorset, D. L., Gilmore, C. J., Jorda, J. L. \& Nicolopoulos, S. (2007). Ultramicroscopy, 107, 462-473.

Dorset, D. L., McCourt, M. P., Li, G. \& Voigt-Martin, I. G. (1998). J. Appl. Cryst. 31, 544-553.

Gemmi, M., Calestani, G. \& Migliori, A. (2002). Adv. Imag. Electron Phys. 123, 311-325.

Gilmore, C. J., Dong, W. \& Dorset, D. L. (2008a). Acta Cryst. A64, 284-294.

Gilmore, C. J., Dong, W. \& Dorset, D. L. (2008b). Acta Cryst. A64, 295-302.

Gramm, F., Baerlocher, Ch., McCusker, L. B., Warrender, S. J., Wright, P. A., Han, B., Hong, S. B., Liu, Z., Ohsuna, T. \& Terasaki, O. (2006). Nature (London), 444, 79-81.

Grosse-Kunstleve, R. W., McCusker, L. B. \& Baerlocher, Ch. (1997). J. Appl. Cryst. 30, 985-995.

Hovmöller, S. (1992). Ultramicroscopy, 41, 121-135.

Koningsveld, H. van, van Bekkum, H. \& Jansen, J. C. (1987). Acta Cryst. B43, 127-132.

Oszlányi, G. \& Sütô, A. (2004). Acta Cryst. A60, 134-141.

Oszlányi, G. \& Sütő, A. (2005). Acta Cryst. A61, 147-152.

Oszlányi, G. \& Sütő, A. (2008). Acta Cryst. A64, 123-134.

Own, C. S., Marks, L. D. \& Sinkler, W. (2006). Acta Cryst. A62, 434443.

Palatinus, L. \& Chapuis, G. (2007). J. Appl. Cryst. 40, 786-790.

Vincent, R. \& Midgley, P. A. (1994). Ultramicroscopy, 53, 271-282.

Weirich, T. E., Portillo, J., Cox, G., Hibst, H. \& Nicolopoulos, S. (2006). Ultramicroscopy, 106, 271-282.

Wessels, T., Baerlocher, Ch., McCusker, L. B. \& David, W. I. F. (2002). Structure Determination from Powder Diffraction Data, edited by W. I. F. David, K. Shankland, L. B. McCusker \& Ch. Baerlocher, pp. 162-178. New York: Oxford University Press.

Zou, X. D. \& Hovmöller, S. (2008). Acta Cryst. A64, 149-160.

Zou, X. D., Sukharev, Y. \& Hovmöller, S. (1993). Ultramicroscopy, 49, $147-158$. 